\title{
A quantitative approach to the study of visual symmetry
}

\author{
PETER G. SZILAGYI and JOHN C. BAIRD \\ Dartmouth College, Hanover, New Hampshire 03755
}

\begin{abstract}
An experiment was conducted to explore the quantitative relationship between "goodness" and the amount of symmetry in visual designs. Subjects arranged items in one, two, and three dimensions in an aesthetically pleasing manner. Each design was analyzed to determine its amount of symmetry, and the percent of subjects who created designs having each possible degree of asymmetry was calculated. Perfect symmetry was the most common condition for all three dimensions. Moreover, the goodness of patterns was inversely related to the quantitative degree of asymmetry.
\end{abstract}

The concept of symmetry has fascinated artists, philosophers, and mathematicians since the ancient Greeks, and it has long been recognized that a symmetric design is visually pleasing. Aristotle believed that "the chief forms of beauty are order and symmetry and definiteness, which the mathematical sciences demonstrate in a special degree" (Ross, 1940 , p. 1078). The notion that symmetrical objects possess desirable qualities has since been a part of many quantitative theories of aesthetic measurement (Birkhoff, 1933; Bosanquet, 1917; Eysenck, 1942).

Around the turn of the century, it was proposed that ocular movement or some similar character of the eye's performance explained the preference for symmetrical shapes. However, Stratton (1906) showed that the eye's behavior alone cannot account for the enjoyment of symmetrical forms. Lacking a physiological theory, many psychologists then turned to the concept of the "good Gestalt." According to Koffka, the term "good" was not clearly defined, but seemed to encompass "such properties as regularity, symmetry, simplicity and others" (1935, p. 110). One reason for this definitional vagueness was the lack of an adequate quantitative theory linking aesthetic pleasure and symmetry (see Eysenck, 1940, 1942).

A major step toward quantifying this relationship was taken by Attneave (1955), who investigated the role of symmetry in the memory for patterns. He found that symmetrical patterns were more accurately reproduced from memory than were asymmetrical patterns only when the symmetrical designs contained less information. ${ }^{1}$ The concept of redundancy of information in a pattern was invoked to explain these results and to provide a unifying interpretation

Reprint requests should be sent to John C. Baird, Department of Psychology, Dartmouth College, Hanover, New Hampshire 03755. of visual preferences (Attneave, 1954, 1955; Michels \& Zusne, 1965). Redundancy, or surplus information, designated by regularities in the forms, was a measurable property which was directly related to the goodness of patterns. Within this framework, a pattern is good because it is redundant or predictable from one of its sections, whereas an unpredictable pattern, lacking redundancy, is a poor one.

More recently, Garner $(1962,1966)$ suggested that the relationship between redundancy and pattern goodness can be viewed in terms of the size of subsets of meaningfully related stimuli. Thus, when perceiving a reference pattern, the observer infers a subset of equivalent patterns, and the goodness of the reference pattern is inversely related to the size of the inferred subset. Garner and Clement (1963) tested this hypothesis and found that good patterns do have small inferred subsets. Subsets are formed by reflections and $90^{\circ}$ rotations of the reference pattern (Garner, 1970). While the goodness of a pattern was often directly related to the number of axes of symmetry, the inverse relationship between the number of alternatives (subsets) and pattern goodness was more generally found. In this approach, symmetry was defined as a reflection across any of four axes (horizontal, vertical, and two diagonals) which reproduced the same pattern. In brief, good patterns are redundant and have few alternatives or equivalent subsets.

Our understanding of what constitutes a good pattern has certainly improved since the days of the "good Gestalt," but knowledge of the quantitative relationship between the amount of symmetry in designs and aesthetic preference is still scant. Amount of symmetry is usually measured by the number of axes through which reflection will reproduce the pattern (Attneave, 1957; Birkhoff, 1933; Garner, 1970). Previous studies, however, have been limited in three important ways: First, they invariably use two-dimensional patterns, usually dots 
(Attneave, 1954, 1957; Garner, 1970; Handel \& Garner, 1966) or square matrices (Schnore \& Partington, 1967). It has been known for some time (Birkhoff, 1933) that symmetry is a characteristic of one-, two- and three-dimensional space. Secondly, recent studies usually provide the stimuli to be judged, rather than allowing the observer to create his own patterns. Such procedures allow for a priori manipulation and analysis of stimulus characteristics, but do not determine the observer's ideal pattern. A person asked to choose between prearranged patterns, none of which are visually pleasing, may base judgments on innumerable factors which the experimenter has not consciously manipulated. Thirdly, in most studies, patterns are designated as either symmetrical or asymmetrical; intermediate degrees of asymmetry are not specified.

In the present work, we have attempted to solve these procedural and analytic limitations by extending the original concepts introduced by Gestalt psychologists. Although the redundancy or the number of equivalent subsets of patterns may accurately predict pattern goodness, it is unlikely that observers consciously evaluate these design characteristics. We believe observers are aware of the symmetry in the patterns and that, therefore, a quantitative analysis relating symmetry and perceived goodness will better reveal design preference from the observer's standpoint. The first limitation was resolved by analyzing the preference for one-, two-, and three-dimensional patterns. Furthermore, we followed Fechner (1876) in allowing subjects to manipulate stimuli, rather than using a technique in which the stimuli were prespecified. ${ }^{2}$ Our procedure employed a design-production task in which ideal patterns were created. The third limitation, namely, the failure to take into account the amount of symmetry in designs, was solved by the use of a new quantitative method. In short, our approach provided a direct test of design preference and the importance of symmetry in pattern goodness.

\section{METHOD}

\section{Subjects}

The subjects were 20 undergraduates ( 17 males and 3 females) enrolled in an introductory psychology course at Dartmouth College.

\section{Materials}

The general procedure consisted of subjects' arranging small square pieces or cubes (design elements) in one, two, or three dimensions. The spaces are illustrated in Figure 1.

For the one- and two-dimensional conditions, the boards were constructed from beige cardboard and the elements were white cardboard squares. The one-dimensional board (Figure 1a) consisted of a row of 18 square cells $(4 \times 4 \mathrm{~cm})$. Eight design elements were used, each the size of one cell. A black dot $(6 \mathrm{~mm}$ in diameter) was placed in the center of each element in order to accentuate the contrast between the elements and the board. (a) ONE DIMENSION

\begin{tabular}{|l|l|l|l|l|l|l|l|l|l|l|l|l|l|l|l|l|l|}
\hline 1 & 2 & 3 & 4 & 5 & 6 & 7 & 8 & 9 & 10 & 11 & 12 & 13 & 14 & 15 & 16 & 17 & 18 \\
\hline
\end{tabular}

(b) TWO DIMENSIONS

\begin{tabular}{|c|c|c|c|c|}
\hline 1 & 2 & 3 & 4 & 5 \\
\hline 6 & 7 & 8 & 9 & 10 \\
\hline 11 & 12 & 13 & 14 & 15 \\
\hline 16 & 17 & 18 & 19 & 20 \\
\hline 21 & 22 & 23 & 24 & 25 \\
\hline
\end{tabular}

(c) THREE DIMENSIONS

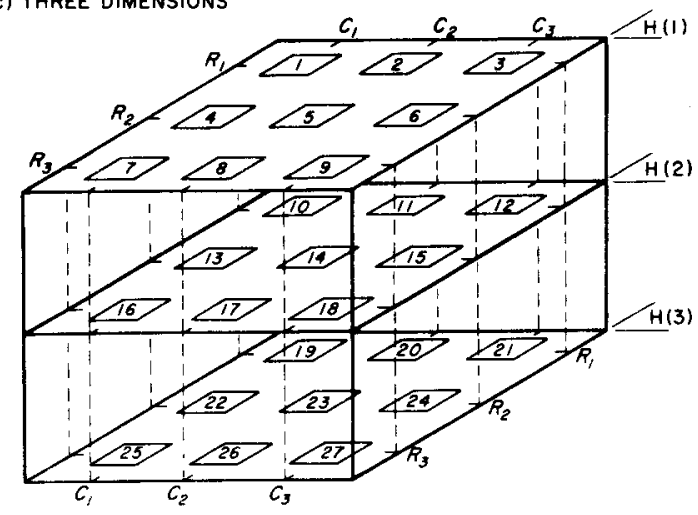

Figure 1. The three displays used in Experiment 1. Eight square elements were arranged in one dimension, 11 in two dimensions, and 12 cubes in three dimensions. The displays are numbered here to clarify the data analysis. The actual displays were not numbered.

The two-dimensional grid (Figure $1 \mathrm{~b}$ ) contained 25 square cells $(5 \times 5 \mathrm{~cm})$ with 11 square design elements.

The three-dimensional display (Figure 1c) consisted of three horizontal planes, each containing 9 square holes, spaced one above the other. The structure was built out of Plexiglas to allow subjects to easily view the horizontal, vertical, and diagonal planes. The design elements were 12 opaque cubes $(4 \times 4 \times 4 \mathrm{~cm})$ which fit exactly into the square holes.

The specific design elements and cells were selected to allow subjects to create many different types of patterns without being constrained by the number of design elements relative to the available cells. The ratio of pieces to cells was held constant at .44 for each of the tasks (i.e., $8 / 18,11 / 25$, and 12/27).

\section{Procedure}

The experiment consisted of three tasks, one for each type of space. Each subject completed all three tasks in random order. The instructions were presented in writing and avoided such words as "symmetry, pattern, design," etc. For the onedimensional task, the subjects were told to arrange the 8 square pieces in a manner that was "visually pleasing." Each piece had to entirely cover one cell. The subjects were required to use all the pieces and were allowed to rearrange them as often as they desired. The two- and three-dimensional tasks were the same, except that 11 pieces were arranged in the 5 by 5 matrix and the 12 cubes were arranged within the three-dimensional structure. At the conclusion of the experiment, the subjects completed a detailed questionnaire concerning the types of strategies they employed. 


\section{RESULTS}

The analysis sought to determine the degree to which subjects created symmetrical and asymmetrical designs in each of the three tasks.

\section{One Dimension}

The analysis involved a mirror-image technique: An imaginary line was drawn between Cell Positions 9 and 10 (Figure la), thus dividing the array into halves. Each position was compared to its corresponding cell in the mirror-image half (e.g., 1 to 18,2 to 17 , and so on), and the entries were subtracted from each other. Since the entries were either 1 or 0 , a perfect match of all cells yielded a resultant of 0 . Such a design would be completely symmetrical, and hence, the degree of asymmetry would 0 . If the two halves matched except for one cell, the design had 1 degree of asymmetry, and so on. For the onedimensional task, the degrees of asymmetry can range from 0 to 8 . However, with an even number of design elements, it was impossible to obtain oddnumbered degrees of asymmetry.

Sixty-five percent of the subjects created designs having perfect symmetry. For comparison purposes, we computed the percentage of different degrees of asymmetry that would occur by chance alone. Four degrees of asymmetry are most probable, and perfect symmetry is a highly unlikely state. In fact, we calculate that the probability of obtaining a perfectly symmetrical pattern is .00288 .

\section{Two Dimensions}

The analysis of the two-dimensional patterns was a generalized version of the mirrror-image technique employed in evaluating the one-dimensional designs. In two dimensions, there are five possible types of symmetry (see Figure 1b): (1) left-right (L-R) sym- metry is found by reflecting the left and right halves of the matrix across an imaginary vertical line passing through the middle column; (2) up-down (U-D) symmetry is a reflection across an imaginary horizontal line passing through the middle row; (3) upper-left-lower-right diagonal symmetry (ULLR) is a reflection across a diagonal line drawn from the upper-left to the lower-right cell; (4) upper-rightlower-left diagonal symmetry (UR-LL) is a reflection across a diagonal line passing through the upper-right and lower-left cells; and (5) rotational (R) symmetry is a $180^{\circ}$ rotation.

Our definition of two-dimensional symmetry differs somewhat from that of Garner (1970), who did not include the rotational case. In the past, other researchers have considered rotational symmetry as a major characteristic of designs. Birkhoff (1933) and Hubbell (1940) defined rotational symmetry about a point as rotation of a polygon (usually in steps of $90^{\circ}$ ). For simplicity, we limited R symmetry to $180^{\circ}$ rotation of the design $\left(90^{\circ}\right.$ rotational symmetry did not occur in most designs).

The analysis compared one-half of the matrix to the other for each of the five types of symmetries. In all cases except the rotational, the middle row was ignored (since it must equal itself) and the remaining two corresponding regions were compared. Since there were 25 cells and 11 design elements, the maximum possible degrees of asymmetry for all cases except the rotational was 10 , obtained when one side was completely filled and the extra element was placed along the axis of symmetry (in this unique case, the degree of $R$ asymmetry was 11 ). The results are presented in Table 1, which gives the percentage of subjects exhibiting each possible degree of asymmetry. The mean percentage is given to the right, alongside the percentage produced by chance. ${ }^{3}$ The middle degrees of asymmetry are most probable,

Table 1

Analysis of Two-Dimensional Designs for Each of Five Types of Symmetry

\begin{tabular}{|c|c|c|c|c|c|c|c|}
\hline \multirow{2}{*}{$\begin{array}{l}\text { Degrees of } \\
\text { Symmetry }\end{array}$} & \multicolumn{5}{|c|}{ Percentages } & \multirow[b]{2}{*}{ Mean } & \multirow{2}{*}{$\begin{array}{c}\text { Chance } \\
(\mathrm{L}-\mathrm{R})\end{array}$} \\
\hline & L-R & U-D & UL-LR & UR-LL & $\mathbf{R}$ & & \\
\hline 0 & 40 & 20 & 25 & 25 & 55 & 33 & .08 \\
\hline 1 & 0 & 0 & 5 & 5 & 0 & 2 & .81 \\
\hline 2 & 25 & 30 & 35 & 35 & 5 & 26 & 3.7 \\
\hline 3 & 5 & 0 & 0 & 0 & 0 & 1 & 10.6 \\
\hline 4 & 0 & 0 & 10 & 10 & 5 & 5 & 19.3 \\
\hline 5 & 10 & 5 & 5 & 10 & 5 & 7 & 24.4 \\
\hline 6 & 10 & 20 & 10 & 5 & 10 & 11 & 21.4 \\
\hline 7 & 5 & 15 & 5 & 5 & 15 & 9 & 13.1 \\
\hline 8 & 5 & 5 & 5 & 5 & 0 & 4 & 5.2 \\
\hline 9 & 0 & 5 & 0 & 0 & 5 & 2 & 1.2 \\
\hline 10 & 0 & 0 & 0 & 0 & 0 & 0 & .1 \\
\hline 11 & & & & & 0 & & \\
\hline
\end{tabular}

Note-The percent of designs exhibiting each of the 11 possible degrees of asymmetry are given along with the mean percentages and the percentages based on chance for $L-R$ symmetry. Data based on 20 subjects. L-R, left-right; U-D, up-down; UL-LR, upper leftlower rught; UR-LL, upper right-lower left; and $R, 180$-deg rotation. 
whereas perfect symmetry is highly unlikely.

The foregoing analysis permits a possible comparison of the strategies employed by subjects in creating different types of symmetries (refer to Table 1). Left-right and rotational symmetries are preferred: $47 \%$ of the subjects indicated on the questionnaire that they focused on one or a couple of aspects of design planning (such as $\mathrm{L}-\mathrm{R}$ and $\mathrm{R}$ symmetry). However, the general tendency toward symmetry is apparent. Forty-five percent of all subjects created designs having one perfect symmetry, $40 \%$ had three, and only $15 \%$ had no perfect symmetries. Under the present experimental conditions, four or five perfect symmetries are not possible from one design.

\section{Three Dimensions}

The analysis of the design in three dimensions requires an examination of each of the planes contained in the space. There are 15 such planes (refer to Figure 1c). There are three horizontal planes $[H(1), H(2), H(3)]$, each consisting of three rows $\left(R_{1}, R_{2}\right.$, and $\left.R_{3}\right)$ and three columns $\left(C_{1}, C_{2}\right.$, and $\left.C_{3}\right)$. There are eight vertical planes: three passing through rows, three passing through columns, and two cross-planes. The cross-planes intersect the three horizontal levels, passing through the diagonals of each level. Thus, $\mathrm{V}\left(\mathrm{X}_{1}\right)$ passes through the upperleft-lower-right diagonals of each diagonal level, and $\mathrm{V}\left(\mathrm{X}_{2}\right)$ intersects the upper-right-lower-left diagonals. There are four diagonal planes, two passing through rows and two through columns. For the former two, $D_{1}(R)$ is the diagonal plane intersecting $R_{1}$ of $H(1)$, $R_{2}$ of $H(2)$, and $R_{3}$ of $H(3)$; and $D_{2}(R)$ passes through $R_{3}$ of $H(1), R_{2}$ of $H(2)$, and $R_{1}$ of $H(3)$. Finally, $D_{1}(C)$ is the diagonal plane passing through $\mathrm{C}_{1}$ of $\mathrm{H}(1)$, $\mathrm{C}_{2}$ of $\mathrm{H}(2)$, and $\mathrm{C}_{3}$ of $\mathrm{H}(3)$; while $\mathrm{D}_{2}(\mathrm{C})$ passes through $\mathrm{C}_{3}$ of $\mathrm{H}(1), \mathrm{C}_{2}$ of $\mathrm{H}(2)$, and $\mathrm{C}_{1}$ of $\mathrm{H}(3)$.

Two analyses were performed. The first determined the number of perfect symmetries within each of the 15 planes. This approach is similar to that used to evaluate the two-dimensional designs, except that here the planes are 3 by 3 matrices. The second analysis compared the horizontal, vertical, and diagonal planes to determine the number of perfect matches. The same standard format was followed in both analyses. The appropriate plane was extracted, reflected, and rotated onto a 3 by 3 matrix such that the numbers representing each cell increased numerically from left to right across rows and down columns (see Figure 1).

The results of the first analysis are summarized in Table 2, in which the percent of perfect symmetries is given for each of the 15 planes. The five types of symmetry are identical to the twodimensional case. As Table 2 reveals, at least $60 \%$ of the subjects created designs having perfect L-R symmetry for all three horizontal planes. More than
Table 2

Analysis of Symmetry for Each of 15 Planes ( 3 by 3 matrices)

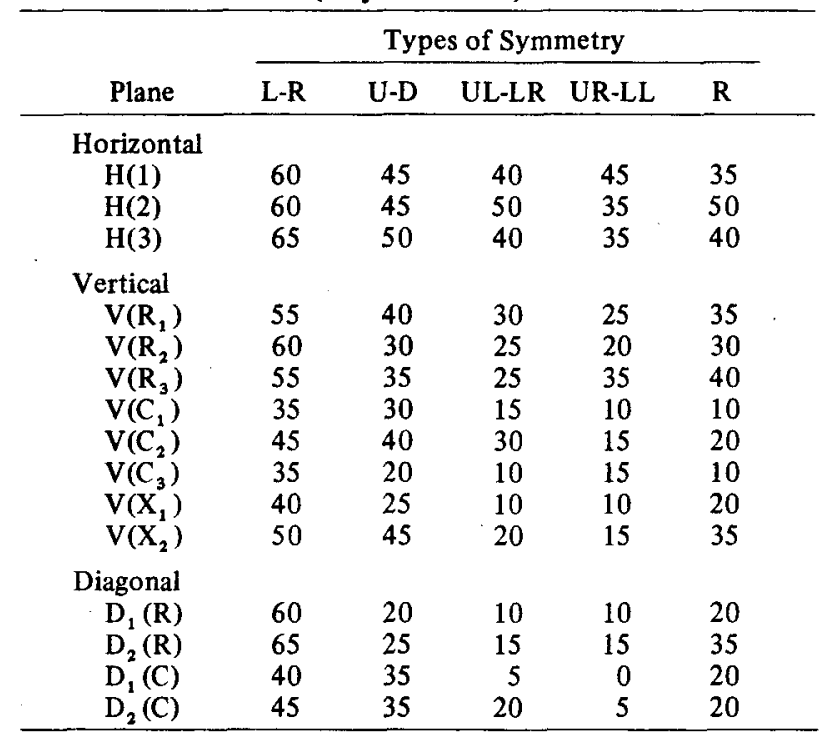

Note-Data entries are the percent of subjects who had perfect symmetry for each of the five types. Data are from 20 subjects. For more detail, see the text. $R$, rows; $C$, columns; and, $X$,crossplane elements.

one-third of the designs had perfect symmetry for the additional four types. Comparing Table 2 to Table 1 , we note that a greater percentage of subjects created perfectly symmetrical designs on all three horizontal planes than on the 5 by 5 grid, for all types of symmetry with the exception of the rotational. As in the two-dimensional case, the percentage of U-D, ULLR, and UR-LL symmetries was approximately equal. A large percentage of subjects created symmetrical designs for all three types of vertical planes. Left-right symmetry was the most popular in each case. In all, $95 \%$ of the subjects created threedimensional designs exhibiting at least one perfect symmetry among the rows $V(R), 95 \%$ among the columns $\mathrm{V}(\mathrm{C})$, and $85 \%$ among the two cross-planes $\mathrm{V}(\mathrm{X})$. The pattern of results for the diagonal planes was similar to the one obtained for the vertical planes.

The second analysis involved a comparison of the cells of one plane to the corresponding cells of another. The percentage of perfect matches was then calculated. Such a match is an indication of symmetry or balance in the three-dimensional design. The results are shown in Table 3. Surprisingly, there were more perfect matches between the vertical planes than the horizontal: $30 \%$ of the designs had identical $V\left(R_{1}\right)$ and $V\left(R_{3}\right)$ cells, while $55 \%$ had perfect matches between the cells on $V\left(C_{1}\right)$ and $V\left(C_{3}\right)$, as well as between the two cross-planes. These results again suggest that creating symmetry over the vertical planes was a critical consideration for many subjects. 
Table 3

Comparison of Neighboring Planes $(x, y)$ to Determine the Percent of Perfect Matches in the Three-Dimensional Case

\begin{tabular}{cccc} 
Plane & \multicolumn{3}{c}{ \% Perfect Matches } \\
\cline { 2 - 3 } $\begin{array}{c}\mathrm{x}=1 \\
\mathrm{y}=2\end{array}$ & $\begin{array}{l}\mathrm{x}=1 \\
\mathrm{y}=3\end{array}$ & $\begin{array}{l}\mathrm{x}=2 \\
\mathrm{y}=3\end{array}$ \\
\hline $\begin{array}{c}\text { Horizontal } \\
\text { H(x)-H(y) }\end{array}$ & 5 & 20 & 5 \\
Vertical & & & \\
$\mathrm{V}\left(\mathrm{R}_{\mathbf{x}}\right)-\mathrm{V}\left(\mathrm{R}_{\mathbf{y}}\right)$ & 5 & 30 & 0 \\
$\mathrm{~V}\left(\mathrm{C}_{\mathbf{x}}\right)-\mathrm{V}\left(\mathrm{C}_{\mathbf{y}}\right)$ & 0 & 55 & 0 \\
$\mathrm{~V}\left(\mathrm{X}_{\mathbf{x}}\right)-\mathrm{V}\left(\mathrm{X}_{\mathbf{y}}\right)$ & 55 & & \\
Diagonal & & & \\
$\mathrm{D}_{\mathbf{x}}(\mathrm{R})-\mathrm{D}_{\mathbf{y}}(\mathrm{R})$ & 35 & & \\
$\mathrm{D}_{\mathbf{x}}(\mathrm{C})-\mathrm{D}_{\mathbf{y}}(\mathrm{C})$ & 60 & \\
\hline
\end{tabular}

Note-Data based on 20 subjects. $R$, rows; $C$, columns; and, $X$, cross-plane elements.

Sixty-five percent had at least one perfect vertical match, $55 \%$ had at least two matches, and $20 \%$ had a perfect match for all three types of vertical planes. Finally, $60 \%$ had identical planes passing through the columns, while one-third of the designs had identical $D_{1}(R)$ and $D_{2}(R)$ planes.

In summary, symmetry was a primary component of most subjects' designs, as the results for all three dimensions reveal. Perfect symmetry was the most favored condition. In addition, over $70 \%$ of all subjects stated on the questionnaire that they actively attempted to create at least one type of symmetry. Many of these subjects also indicated a desire to maximize several different types of symmetry. These results strongly support the hypothesis that observers are aware of the symmetry in designs, and that symmetry is a primary attribute of good patterns.

Given these results, we felt it worthwhile to evaluate the phenomenon in a more general context with more subjects. This was done in a second experiment with 55 subjects who created designs in one and two dimensions. The density of items was increased to .64 (no. items/no. of cells) for two dimensions and to .73 for one dimension. The results agreed substantially with those just reported. For example, in two dimensions, $80 \%$ of the subjects had designs with at least one perfect symmetry: $42 \%$ had exactly one, $13 \%$ had two, and $25 \%$ had five.

\section{DISCUSSION}

In investigating aesthetic preference, psychologists have often classified patterns into types which can be controlled in the laboratory. Several such classifications have recently been developed. Researchers have investigated the complexity of stimuli (Attneave, 1957; Berlyne, 1958), the amount of information or redundancy in patterns (Attneave, 1954, 1955; Klemmer \& Frick, 1953), and the number of alternatives that patterns possess (Garner, 1962, 1966, 1970; Garner \& Clement, 1963). In many of these studies, the importance of symmetry has been noted, but not adequately investigated. The present quantitative analysis relating the amount of symmetry in a pattern and its goodness provided a direct and simple test of aesthetic preference. Although it is probably true that a good pattern is redundant and has few equivalent subsets, an observer may not be cognizant of these aspects of a stimulus display. On the other hand, most of our observers were aware of the symmetry in designs, as indicated by their responses on the questionnaire administered after Experiment 1. We feel that symmetry is a primary, and perhaps the most important, characteristic of designs which contributes to the goodness of the patterns.

We have defined a "good pattern" as one which is often created in a design-production task, whereas a poor pattern is one which is not commonly constructed. Thus, the goodness of a pattern is operationally defined by the percent of subjects who create such a pattern. Several conclusions can be reached relating degree of asymmetry and pattern goodness. First, the best pattern is one which is perfectly symmetrical, or has 0 degree of asymmetry. Secondly, the goodness of a pattern drops off rapidly with increasing degrees, as seen by the smaller number of subjects who created such designs. Thirdly, this phenomenon occurs for one-, two-, and threedimensional displays. Since it has also been observed for dot patterns (Garner, 1970) and other shapes (Hubbell, 1940), it is safe to conclude that we are dealing with a general phenomenon.

While many subjects focused upon the type of symmetry in their two- and three-dimensional designs, an almost equal percentage created patterns having more than one perfect symmetry. These results suggest two types of planning strategies-one attempts to maximize a particular type of symmetry, perhaps at the expense of others; the second strategy attempts to create several types of perfect symmetry. This conclusion is clearly speculative, but does suggest that the strategies employed by subjects in a design-production task should be more closely investigated, something that cannot be done when subjects are asked to rate the goodness of patterns created by the experimenter. Previous work suggests, however, that ratings of goodness would correlate highly with the measures based on production data (at least for these elementary tasks).

Patterns are used as stimuli in all sorts of psychological experiments, and while an aesthetic factor is occasionally noted as a possible variable, aesthetic preference is not usually controlled. By either predetermining or analyzing (in a production task) the degree of asymmetry in such stimuli, we now have 
a quantitative method to determine this aesthetic factor. For example, one study on the planning of an ideal town used three matrices identical to those in Experiment 2 (Baird, Degerman, Paris, \& Noma, 1972). An analysis of the degrees of asymmetry in plans produced in such experiments would allow one to determine the extent to which aesthetic factors may have influenced the results.

These results can also be applied to parametric studies of visual perception. For example, King, Meyer, Tangney, and Biederman (1976) reported a perceptual bias towards symmetry in judging the shape of a rotated object. However, the "asymmetrical figures" they employed did possess a great deal of symmetry. A quantitative measure of the various types of symmetry would clearly be useful in studying the perceptual or memory bias towards truly symmetrical forms (see also, Boswell, 1976). The present technique for quantifying amount of symmetry (asymmetry), as well as the results on the perceived goodness of forms, could be profitably used in such parametric studies.

\section{REFERENCES}

Adams, O. S., Fitts, P. M., Rappaport, M., \& Weinstein, M. Relations among some measures of pattern discriminability. Journal of Experimental Psychology, 1954, 48, 81-88.

AtTNEave, F. Some informational aspects of visual perception. Psychological Review, 1954, 61, 183-193.

AttNeave, F. Symmetry, information and memory for patterns. American Journal of Psychology, 1955, 68, 209-222.

Attneave, F. Physical determinants of the judged complexity of shapes. Journal of Experimental Psychology, 1957, 53, 221-227.

Baird, J. C., Degerman, R., Paris, R., \& Noma, E. Student planning of town configuration. Environment and Behavior, 1972, 4, 159-188.

BERLYNE, D. E. The influence of complexity and novelty in visual figures on orienting responses. Journal of Experimental Psychology, 1958, 55, 289-296.

Birkhoff, G. D. Aesthetic measure. Cambridge: Harvard University Press, 1933.

BosANQUET, B. A history of aesthetics. London: Allen \& Unwin, 1917.

BoswelL, S. L. Young children's processing of asymmetrical and symmetrical patterns. Joumal of Experimental Child Psychology, 1976, 22, 309-318.

EYSENCK, $H$. J. The general factor in aesthetic judgments. British Journal of Psychology, 1940, 31, 94-102.

EYSENCK, H. J. The experimental study of the "good Gestalt"-a new approach. Psychological Review, 1942, 49, 344-364.
Fechner, G. T. Vorschule der Aesthetik. Leipzig: Brietkopf \& Hartel, 1876.

French, R. S. The discrimination of dot patterns as a function of number and average separation of dots. Journal of Experimental Psychology, 1953, 46, 1-9.

French, R. S. Pattern recognition in the presence of visual noise. Journal of Experimental Psychology, 1954, 47, 27-31.

GARNER, W. R. Uncertainty and structure as psychological concepts. New York: Wiley, 1962.

Garner, W. R. To perceive is to know. American Psychologist, 1966, 21, 11-19.

Garner, W. R. Good patterns have few alternatives. American Scientist, 1970, 58, 34-42.

Garner, W. R., \& Clement, D. E. Goodness of pattern and pattern uncertainty. Journal of Verbal Learning \& Behavior. $1963,2,446.452$.

HANDEL, S., \& Garner, W. R. The structure of visual pattern associates and pattern goodness. Perception \& Psychophysics, 1966, 1, 33-38.

Hubbell, M. B. Configurational properties considered 'good' by naive subjects. American Journal of Psychology, 1940, 53, 46-69.

King, M., MeYer, G. E., Tangney, J., \& Biederman, I. Shape constancy and a perceptual bias towards symmetry. Perception \& Psychophysics, 1976, 19, 129.136.

Klemmer, F. T., \& Frick, F. C. Assimilation of information from dot and matrix patterns. Journal of Experimental Psychology, 1953, 45, 15-19.

KofFKA, H. Principles of Gestalt psychology. London: Kegan Paul, 1935.

Michels, K. M., \& Zusne, L. Metrics of visual form. Psychological Bulletin, 1965, 63, 74-86.

Ross, W. D. (Ed.) Metaphysica. In The works of Aristotle (Vol. VIII). Oxford: Clarendon Press, 1940.

Schnore, M. M., \& PARtington, J. T. Immediate memory for visual patterns: Symmetry and amount of information. Psychonomic Science, 1967, 8, 421-422.

Stratron, G. M. Symmetry, linear illusions, and the movements of the eye. Psychological Review, 1906, 13, 81-96.

\section{NOTES}

1. The role of symmetry in memory and discrimination tasks has been further studied, with similar results, by French (1953, 1954), Adams, Fitts, Rappaport, and Weinstein (1954), and Schnore and Partington (1967).

2. Hubbell (1940) also recognized the importance of active subject participation in the study of "good figures." $\mathrm{He}$ had observers change two-dimensional figures in order to create a "better" figure, and then analyzed the types of changes made.

3. We thank Lauren Tanny and Ann Lucchini for deriving a general formula to calculate the probability of different degrees of asymmetry.

(Received for publication March 31, 1977; revision accepted June 23,1977 .) 